\title{
Time-dependent density functional theory applied to x-ray absorption spectroscopy
}

\author{
Oana Bunău* \\ IMPMC, CNRS/UPMC, 4 place Jussieu, 75005 Paris, France and Institut Néel, CNRS/UJF, 25 rue des Martyrs, \\ BP 166, 38042 Grenoble cedex 9, France \\ Yves Joly \\ Institut Néel, CNRS/UJF, 25 rue des Martyrs, BP 166, 38042 Grenoble cedex 9, France
}

(Received 23 December 2011; published 12 April 2012)

\begin{abstract}
We report the implementation of a time-dependent density functional theory (TDDFT) method in the adiabatic limit (TDLDA) of the calculation of x-ray absorption spectroscopy. We show results for various choices for the exchange-correlation kernel, in particular the absorption spectra at the $L_{2,3}$ edges of the $3 d$ elements. We equally present a detailed study of the method's limitations and range of applicability. We found that TDDFT calculations should be performed fully relativistically even for nonmagnetic materials. We conclude that an accurate TDDFT description of $\mathrm{x}$-ray absorption should include the core hole effects. The local (both in space and time) TDDFT kernels that are currently used in the description of extended systems do not meet this criterion.
\end{abstract}

DOI: 10.1103/PhysRevB.85.155121

PACS number(s): 78.70.Dm, 31.15.A-

\section{INTRODUCTION}

$\mathrm{X}$-ray absorption spectroscopy is a very powerful tool in material science, as it probes the electronic and geometrical structures around the absorbing atom. For such spectroscopy, the available first-principles descriptions and calculation methods are rather behind the experiments in terms of performance and accuracy. Among these, the most common methods are the density functional theory (DFT) based, onebody calculations. ${ }^{1-7}$ Although they describe successfully the delocalized edges (i.e., $K$ edges), they are less reliable with partly localized or atomic edges ( $L_{23}$ edges of transition elements, $M_{45}$ edges of rare earths). In particular, DFT techniques fail to describe the many-body effects such as the multiplet structures ${ }^{8}$ or the relative $L_{3} / L_{2}$ intensities of the absorption spectra of the $3 d$ elements (the so-called branching ratio anomaly ${ }^{9}$ ). Consequently, the breakdown of the single-particle picture suggests the urge of including some many-body effects in the description of the $\mathrm{x}$-ray absorption spectroscopy.

The first attempts to bring calculations beyond the onebody approximation belong to Zangwill and Soven ${ }^{10}$ and Zaanen et al. ${ }^{11}$ The former treat from first principles the local-fields effect in the photoabsorption spectra of rare gases, within linear response and using atomic screening. The latter use a two Green-function model to explain the branching ratio anomaly at the $L_{2,3}$ edges of the early $3 d$ transition metals. These two pioneering pieces of work open the way for the modern first-principles calculations of x-ray absorption in extended systems beyond the one-body approximation: the multichannel method, ${ }^{12-14}$ the Bethe Salpeter equation $(\mathrm{BSE})^{15,16}$ and the time-dependent density functional theory (TDDFT) based calculations. ${ }^{17-21}$ Among these, TDDFT is particularly convenient in terms of simplicity and calculation time. In this paper we focus on the application of the TDDFT to the $\mathrm{x}$-ray absorption frequency range, as we feel that the existing literature is not conclusive enough on the subject. We therefore propose our own implementation of the TDDFT method in the adiabatic approximation (TDLDA), as well as an extensive study of its range of applicability. Note that results of non-muffin-tin effects in TDDFT will be published elsewhere without being explicit on the method. In this paper we describe the details of the implementation of our TDDFT procedure and compare it to the other results in the literature.

The structure of this paper is as follows. The second section is dedicated to the application of the TDDFT to the resonant case and contains a detailed description of the several choices of the exchange correlation kernel. The third section describes the implementation of the TDDFT calculation within the FDMNES (Finite Differences Method Near Edge Structures) code. ${ }^{1}$ The fourth and fifth sections contain the extended discussion of our results and the conclusion, respectively.

\section{TDDFT FOR X-RAY ABSORPTION}

In the spirit of Zangwill and Soven, ${ }^{10}$ Runge and Gross propose a more general, though related, method to calculate the absorption cross section: the time-dependent density functional theory (TDDFT). ${ }^{22}$ Although the TDDFT has quickly become popular for the quantum chemistry studies, its application to condensed-matter studies is more recent. ${ }^{23,24}$ The sophisticated, often parametrized exchange-correlation functionals of quantum chemistry lose their special behavior and are no longer useful in extended systems. ${ }^{25}$ Therefore exchange-correlation functionals in condensed-matter studies are based on the uniform electron gas model, whose limitations make TDDFT less successful for solids than it is for molecules. Indeed, for the description of delocalized excitations, TDLDA reduces to DFT essentially.

Contrary to standard DFT, TDDFT includes the time dependence, a feature that is essential for spectroscopic calculations. In TDDFT, the coupling of the electromagnetic field to the sample is treated as a perturbation. Perturbation theory can be applied in the case of common beam intensities (the third generation synchrotrons) and one can restrain the TDDFT to the linear response (LR) regime. Note that perturbation theory is no longer valid when one deals with laser pulses or X-FELs, i.e., whose corresponding fields are of the same order of magnitude with the electric field inside the atom. This work 
only deals with the LR regime, where ${ }^{10}$

$$
\begin{aligned}
\sigma(\omega)= & -\frac{4 \pi \omega}{c} \sum_{\sigma \sigma^{\prime}} \int d^{3} r \int d^{3} r^{\prime} \\
& \times \hat{O}^{\dagger}\left(\omega, \vec{r}^{\prime}\right) \operatorname{Im} \chi^{\sigma \sigma^{\prime}}\left(\omega, \vec{r}, \vec{r}^{\prime}\right) \hat{O}(\omega, \vec{r})
\end{aligned}
$$

Here $\omega$ is the photon energy, $\sigma$ is the spin index, $\sigma(\omega)$ is the absorption cross section, $\chi$ is the linear-response function, and $\hat{O}$ is the external field operator. Starting from Eq. (1), we shall be employing the atomic (Hartree) unit system all through this paper.

The standard DFT calculations assume $\hat{\chi}=\hat{\chi}_{0}$, i.e., use the ground-state response function $\chi_{0}$ of the noninteracting system. The spirit of the LR-TDDFT is to include the manybody effects within the response function:

$$
\hat{\chi}=\hat{\chi}_{0}+\hat{\chi}_{0} \hat{K} \hat{\chi}
$$

where $\chi$ is the response function that accounts for the manybody corrections. Ideally, the integral kernel $K$ should describe the many-body effects, including the interaction with the core hole. Nonetheless, as no exact analytical form of the exchange correlation is available, one has to assume the form of $K$. The physics one may describe and the accuracy of the description are tributary to the choice of this ansatz.

In the TDDFT approach, the many-body effects associated to the excitation of a core electron are described by an effective single-particle picture of these excitations. To understand this, please note that in the following we will be using the KohnSham orbitals as a basis of the expansion of different quantities (kernel, response functions). Hence we need a ground-state DFT calculation to obtain the basis functions for the TDDFT.

(a) Notations. In the following we will adapt the TDDFT formalism to the $\mathrm{X}$-ray absorption frequency range. We proceed by introducing the notations for the wave functions (spinorbit coupling included). The initial, occupied states (index $g$ ) corresponding to the resonant transition are

$$
\phi_{g}(\vec{r})=\sum_{\sigma} \phi_{g}^{\sigma}(\vec{r}) \zeta_{\sigma}=\sum_{\sigma} c_{\Lambda_{g}^{\sigma}} b_{g}(r) Y_{\Lambda_{g}^{\sigma}}(\hat{r}) \zeta_{\sigma},
$$

where $\Lambda_{g}^{\sigma}=\left(l_{g}, m_{g}+\frac{1}{2}-\sigma, \sigma\right)$ is the set of quantum numbers characterizing the initial states and $\zeta_{\sigma}$ is the spin eigenfunction. One can see that each initial state $\phi_{g}(\vec{r})$ is a sum of the two spin contributions, weighted by the Clebsch-Gordon coefficients $c_{\Lambda_{g}^{\sigma}} . b_{g}(r)$ is the radial wave function, which in practice only depends on the edge corresponding to the initial state $g . Y(\hat{r})$ stands for the complex spherical harmonics.

In the Dirac formalism, after eliminating the high-energy solutions, the final-state wave function can be written in its exact form as ${ }^{26}$

$$
\Psi_{f}(\vec{r}, E)=\sum_{\sigma} \sum_{\Lambda_{s}} a_{\Lambda_{s}}^{f}(E) \Psi_{\Lambda_{\sigma}}^{s}(\vec{r}, E) \zeta_{\sigma} .
$$

When fully relativistic, the eigenfunctions are the sum of two solutions sharing the same $l$ quantum number but different $m .^{27,28} s$ is the index over the solution and by convention we take it either $\frac{1}{2}$ or $-\frac{1}{2}$, i.e., the same values as for the spin projection $\sigma . \Lambda_{s}=\left(l, m+\frac{1}{2}-s, s\right)$ and $\Lambda_{\sigma}=\left(l, m+\frac{1}{2}-\sigma, \sigma\right)$ are sets of quantum numbers describing the final state. $a_{\Lambda_{s}}^{f}(E)$ is the multiple-scattering amplitude of the $\Lambda_{\sigma}$ contribution to the $s$ component of the final-state wave-function:

$$
\Psi_{\Lambda_{\sigma}}^{s}(\vec{r}, E)=b_{\Lambda_{\sigma}}^{s}(r, E) Y_{\Lambda_{\sigma}}(\hat{r}),
$$

where $b_{\Lambda_{\sigma}}^{s}(r, E)$ is the spin and orbital dependent radial wave function. Note that in Eq. (4) the summation over $\Lambda_{s}$ implies

$$
\sum_{\Lambda_{s}} \equiv \sum_{l m s}
$$

with $m$ obeying the constraint $-l \leqslant m+\frac{1}{2}-s \leqslant l$. A similar remark can be made on the $\Lambda_{\sigma}$ summation.

(b) Ground-state susceptibility. For a noninteracting system and in the spherical harmonics representation, the fully relativistic Adler-Wiser equation ${ }^{24}$ reads

$$
\begin{aligned}
\chi_{0}{ }^{\sigma \sigma^{\prime}}\left(\vec{r}, \vec{r}^{\prime}, \omega\right)= & \frac{1}{\pi} \lim _{\epsilon \rightarrow 0} \sum_{g} \int_{E_{F}}^{\infty} d E \\
& \times \sum_{f} \frac{\phi_{g}^{\sigma}(\vec{r}) \Psi_{f}^{\dagger}(\vec{r}, E) \Psi_{f}\left(\vec{r}^{\prime}, E\right) \phi_{g}^{\sigma^{\prime} \dagger}\left(\vec{r}^{\prime}\right)}{\omega-\left(E-E_{g}\right)+i \epsilon},
\end{aligned}
$$

where $f$ are the final states of the same energy $E$. We stress the fact that the energies $\omega$ and $E$ are expressed into two different scales: the former describes the photon, whereas the latter belongs to the photoelectron. $E_{g}$ is the Kohn-Sham energy of the $g$ state, i.e., the expectation value of the effective-single-particle Kohn-Sham Hamiltonian (as given by DFT local density approximation) for this particular state,

$$
E_{g}=\frac{\left\langle\phi_{g}\left|\mathcal{H}_{\mathrm{LDA}}\right| \phi_{g}\right\rangle}{\left\langle\phi_{g} \mid \phi_{g}\right\rangle}
$$

The optical theorem ${ }^{29}$ connects the amplitudes $a_{\Lambda_{s}}^{f}(E)$ and $a_{\Lambda_{s}^{\prime}}^{f}(E)$ and the multiple-scattering matrix $\tau_{\Lambda_{s} \Lambda_{s}^{\prime}}(E)$ of the multiple-scattering theory (MST):

$$
\sum_{f} a_{\Lambda_{s}^{\prime}}^{f}(E) a_{\Lambda_{s}}^{f *}(E)=-\operatorname{Im} \tau_{\Lambda_{s} \Lambda_{s}^{\prime}}(E) .
$$

By exploiting Eqs. (4) and (9), Eq. (7) can be simplified to

$$
\begin{aligned}
\chi_{0}{ }^{\sigma \sigma^{\prime}}\left(\vec{r}, \vec{r}^{\prime}, \omega\right) \\
=-\frac{1}{\pi} \sum_{g g^{\prime}} \delta_{g g^{\prime}} \sum_{\Lambda_{s} \Lambda_{s}^{\prime}} \lim _{\epsilon \rightarrow 0} \int_{E_{F}}^{\infty} d E \\
\quad \times \frac{\phi_{g}^{\sigma}(\vec{r}) \Psi_{\Lambda_{\sigma} s^{\dagger}}(r, E) \operatorname{Im} \tau_{\Lambda_{s} \Lambda_{s}^{\prime}}(E) \Psi_{\Lambda_{\sigma}^{s^{\prime}}}\left(\vec{r}^{\prime}, E\right) \phi_{g^{\prime}}^{\sigma^{\prime} \dagger}\left(\vec{r}^{\prime}\right)}{\omega-\left(E-E_{g}\right)+i \epsilon},
\end{aligned}
$$

where we introduced the explicit dependence on the initial states $g$. We stress the fact that the Adler-Wiser equation ${ }^{24}$ normally involves all the occupied and unoccupied $(E, f)$ states. Nevertheless, in the resonant case, and thus at energies beyond the optical spectrum, one can assume that the only significant contribution is brought in by the levels whose energy $E-E_{g}$ is close to the photon energy $\omega$, i.e., the concerned core levels indexed by $g$ [see Eq. (10)]. Quantitatively, this is an extremely sound simplification and does not introduce errors. ${ }^{30} \chi_{0}$ is diagonal over the initial states $g$, meaning that in a one-particle picture the different channels available to the 
electronic transition do not mix. Note that $\chi_{0}$ involves only ground-state quantities.

In the following we shall exploit the formalism developed by Schwitalla and Ebert in Ref. 17. We are interested in a spherical harmonics representation for $\chi_{0}$. Consequently, we are considering an expansion of the following kind:

$$
\begin{aligned}
\chi_{0}{ }^{\sigma \sigma^{\prime}}\left(\vec{r}, \vec{r}^{\prime}, \omega\right) \\
=\sum_{g g^{\prime}} \delta_{g g^{\prime}} \phi_{g}^{\sigma}(\vec{r}) \phi_{g^{\prime}}^{\sigma^{\prime} \dagger}\left(\vec{r}^{\prime}\right) \sum_{\Lambda_{s} \Lambda_{s}^{\prime}} \tilde{\chi}_{0}^{\sigma g^{\prime}, \Lambda_{s} \Lambda_{s}^{\prime}}(\omega) \\
\quad \times \Psi_{\Lambda_{\sigma} \dagger}^{s \dagger}\left(\vec{r}, \omega+E_{g}\right) \Psi_{\Lambda_{\sigma}^{\prime}}^{s^{\prime}}\left(\vec{r}^{\prime}, \omega+E_{g^{\prime}}\right)
\end{aligned}
$$

as if we were projecting $\chi_{0}$ on the set formed by the initial-state wave functions $\phi$ and the final-state ones $\Psi$. The energy dependence of $\Psi$ is bothersome and needs to be counterbalanced by considering an energy dependence in the very form of $\tilde{\chi}_{0} \sigma \sigma_{g^{\prime}, \Lambda_{s} \Lambda_{s}^{\prime}}$. We suppose that a derivation similar to Eq. (11) is valid for $\tilde{\chi}$.

(c) Connection with the multiple-scattering amplitude. We are trying to establish the connection between $\chi$ and $\tau$, the multiple-scattering amplitude of the MST. Let us investigate the similarities between the results of the LR theory and the MST for the absorption cross section. We recall that in the MST and in the complex spherical harmonics basis ${ }^{26}$

$$
\begin{aligned}
\sigma(\omega)= & -\frac{4 \pi \omega}{c} \int d^{3} r \int d^{3} r^{\prime} \sum_{\sigma \sigma^{\prime}} \sum_{g g^{\prime}} \sum_{\Lambda_{s} \Lambda_{s}^{\prime}} \\
& \times O^{*}(\omega, \vec{r}) b_{\Lambda_{\sigma}}^{s}(r) Y_{\Lambda_{\sigma}}^{*}(\hat{r}) \phi_{g}^{\sigma}(\vec{r}) \operatorname{Im} \tau_{\Lambda_{s} \Lambda_{s}^{\prime}} \\
& \times O^{*}\left(\omega, \vec{r}^{\prime}\right) b_{\Lambda_{\sigma}^{s^{\prime}}}^{s^{\prime}}\left(r^{\prime}\right) Y_{\Lambda_{\sigma}^{\prime}}\left(\hat{r}^{\prime}\right) \phi_{g^{\prime}}^{\sigma^{\prime} \dagger}\left(\vec{r}^{\prime}\right) .
\end{aligned}
$$

One can treat the projection of the susceptibility $\tilde{\chi}$ and the multiple-scattering amplitudes $\tau$ on equal footing:

$$
\tilde{\chi}_{0}^{\sigma \sigma, \Lambda_{s} \Lambda_{s}^{\prime}} \equiv \tau_{\Lambda_{s} \Lambda_{s}^{\prime}} \text {. }
$$

The equivalence is easy to see if one compares Eq. (12) to the definition of $\tilde{\chi_{0}}$ in Eq. (11). This is a remarkable result and it was first suggested by Schwitalla and Ebert. ${ }^{17}$ We stress that the equivalence no longer holds if $\tau$ is issued from a potential that takes complex values. More particularly, the Green function describing the scattering process is required not to have any other solution than the regular one, or otherwise Eq. (13) breaks down. Let

$$
\begin{aligned}
\tilde{\chi}_{0 g g^{\prime}, \Lambda_{s} \Lambda_{s}^{\prime}}^{\sigma \sigma^{\prime}}(\omega)= & -\delta_{g g^{\prime}} \int_{E_{F}}^{\infty} \frac{d E}{\pi} \frac{\operatorname{Im} \tau_{\Lambda_{s} \Lambda_{s}^{\prime}}(E)}{\omega-\left(E-E_{g}\right)+i \epsilon} \\
& \times \frac{Z_{g^{\prime} \Lambda_{\sigma}^{\prime}}^{s^{\prime}}(E) Z_{g \Lambda_{\sigma}}^{s}(E)}{Z_{g^{\prime} \Lambda_{\sigma}^{\prime}}^{s^{\prime}}\left(\omega+E_{g^{\prime}}\right) Z_{g \Lambda_{\sigma}}^{s}\left(\omega+E_{g}\right)},
\end{aligned}
$$

an equivalent form of the equivalence in Eq. (13), where

$$
Z_{g \Lambda_{\sigma}}^{s}(E)=\int_{0}^{R} d r r^{2} b_{g}(r) b_{\Lambda_{\sigma}}^{s}(r, E)
$$

is some energy-dependent function that is supposed to account for the energy modulations in $\Psi$. Note that the $Z$ normalization procedure in Eq. (14) is reasonable as long as the energy dependence in $Z$, and consequently in the radial final-state wave functions, is weak. We stress the fact that this is the only approximation introduced by the method and otherwise the expansion in Eq. (14) would be exact. The necessity of introducing Eq. (14) is as follows. $\tilde{\chi}_{0}$ depends on the photon energy $\omega$, whereas $\tau$ depends on the photoelectron's energy $E$. Equation (14) is the bridge between the two quantities.

(d) Kernel. In the $|r, \sigma\rangle$ representation Eq. (2) rewrites as

$$
\begin{aligned}
\chi^{\sigma \sigma^{\prime}}\left(\vec{r}, \vec{r}^{\prime}, \omega\right) \\
=\chi_{0}^{\sigma \sigma^{\prime}}\left(\vec{r}, \vec{r}^{\prime}, \omega\right)+\sum_{\sigma^{\prime \prime} \sigma^{\prime \prime \prime}} \int d^{3} r^{\prime \prime} \int d^{3} r^{\prime \prime \prime} \\
\quad \times \chi_{0}^{\sigma \sigma^{\prime \prime}}\left(\vec{r}, \vec{r}^{\prime \prime}, \omega\right) K^{\sigma^{\prime \prime} \sigma^{\prime \prime \prime}}\left(\vec{r}^{\prime \prime}, \vec{r}^{\prime \prime \prime}\right) \chi^{\sigma^{\prime \prime \prime} \sigma^{\prime}}\left(\vec{r}^{\prime \prime \prime}, \vec{r}^{\prime}, \omega\right) .
\end{aligned}
$$

To avoid the solving of the integral equation (16), we project the operatorial Dyson-like equation (2) on the basis introduced in Eq. (11):

$$
\begin{aligned}
\tilde{\chi}_{g g^{\prime}, \Lambda_{s} \Lambda_{s}^{\prime}}^{\sigma \sigma^{\prime}}(\omega)= & \tilde{\chi}_{0} \sigma \sigma_{g}, \Lambda_{s} \Lambda_{s}^{\prime} \\
& \times \sum_{g^{\prime \prime \prime}} \sum_{\sigma^{\prime \prime} \sigma^{\prime \prime \prime}} \sum_{\Lambda_{s}^{\prime \prime} \Lambda_{s}^{\prime \prime \prime}}{ }^{\sigma \prime \prime} \Lambda_{s} \Lambda_{s}^{\prime \prime}(\omega) \tilde{K}_{g g^{\prime \prime \prime}, \Lambda_{s}^{\prime \prime} \Lambda_{s}^{\prime \prime \prime}}^{\sigma^{\prime \prime \prime}}(\omega) \tilde{\chi}_{g^{\prime \prime \prime} g^{\prime}, \Lambda_{s}^{\prime \prime \prime} \Lambda_{s}^{\prime}}^{\sigma^{\prime \prime \prime}}(\omega),
\end{aligned}
$$

where we have used $\tilde{\chi_{0}}{ }_{g g^{\prime}, \Lambda_{s} \Lambda_{s}^{\prime}}^{\sigma \sigma^{\prime}}(\omega)=\tilde{\chi_{0}} \tilde{g g}^{\sigma \sigma^{\prime}, \Lambda_{s} \Lambda_{s}^{\prime}}(\omega) \delta_{g g^{\prime}}$. We are interested in $\tilde{K}$, the projection of the kernel on the same basis functions as in Eq. (11). We plug into Eq. (16) the expansions of type (11) for both $\chi_{0}$ and $\chi$ and identify with Eq. (17). One obtains

$$
\begin{aligned}
\tilde{K}_{g g^{\prime}, \Lambda_{s} \Lambda_{s}^{\prime}}^{\sigma \sigma^{\prime}}(\omega) & \\
= & \int d^{3} r \int d^{3} r^{\prime} K^{\sigma \sigma^{\prime}}\left(\vec{r}, \vec{r}^{\prime}, \omega\right) \phi_{g}^{\sigma \dagger}(\vec{r}) \Psi_{\Lambda_{\sigma}}^{s}\left(\vec{r}, \omega+E_{g}\right) \\
& \times \phi_{g^{\prime}}^{\sigma^{\prime}}\left(\vec{r}^{\prime}\right) \Psi_{\Lambda_{\sigma}^{\prime}}^{s^{\prime} \dagger}\left(\vec{r}^{\prime}, \omega+E_{g^{\prime}}\right) .
\end{aligned}
$$

As a technical detail, note the difference with Eq. (11) in terms of Hermitian conjugate factors. In this sense, the form of $\tilde{K}$ both in Ref. 17 [Eq. (11)] and in Ref. 21 [Eq. (26)] is not general (but nevertheless correct for kernels taking real values). In its most general form, the TDDFT kernel can be split into a classical Coulomb (Hartree) term $f_{H}$ and an exchange-correlation contribution $f_{x c}$ :

$$
K^{\sigma \sigma^{\prime}}\left(\vec{r}, \vec{r}^{\prime}, \omega\right)=f_{H}^{\sigma \sigma^{\prime}}\left(\vec{r}, \vec{r}^{\prime}\right)+f_{x c}^{\sigma \sigma^{\prime}}\left(\vec{r}, \vec{r}^{\prime}, \omega\right),
$$

where $f_{H}$ is local and spin independent:

$$
f_{H}^{\sigma \sigma^{\prime}}\left(\vec{r}, \vec{r}^{\prime}\right)=\frac{1}{\left|\vec{r}-\vec{r}^{\prime}\right|} .
$$

(e) Dyson equation. TDDFT provides a calculation recipe to include the many-body interactions, to an extent dictated by the kernel, into the response function. The renormalization in Eq. (2) is actually a matrix equation of the form

$$
\hat{\chi}=\left(\hat{1}-\hat{\chi}_{0} \hat{K}\right)^{-1} \hat{\chi}_{0} \text {. }
$$

While $\tilde{\chi}_{0}$ is diagonal over the initial states $g$, it is not the case for the kernel $\tilde{K}$. Consequently, the many-body response function $\tilde{\chi}$ may mix different initial states. This effect is to be seen on the spectra if one calculates two edges that are close in energy (less than $\approx 50 \mathrm{eV}$ of difference), such as the $L_{2,3}$ edges of the $3 d$ elements. In this case, the overlap between the matrix elements of the real parts of $\tilde{\chi}_{0} \mathrm{~s}$ (Fig. 1) causes a transfer of spectral weight in the imaginary parts of $\tilde{\chi}$. Subsequently, 


\section{$\operatorname{Re} \chi_{0}$}

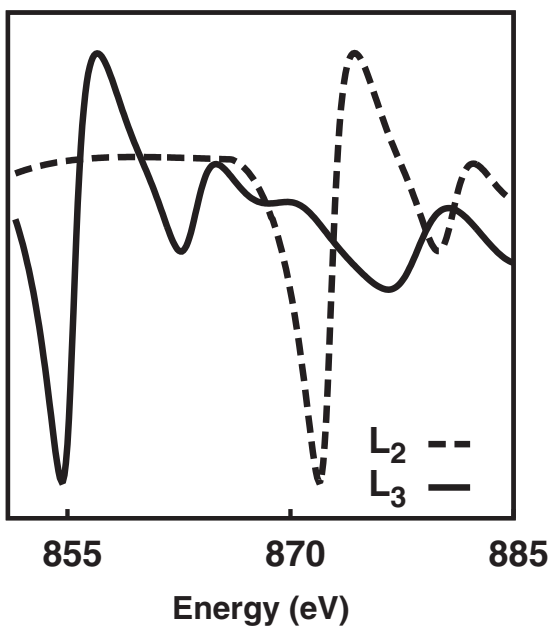

$\operatorname{Im} \chi_{0}$

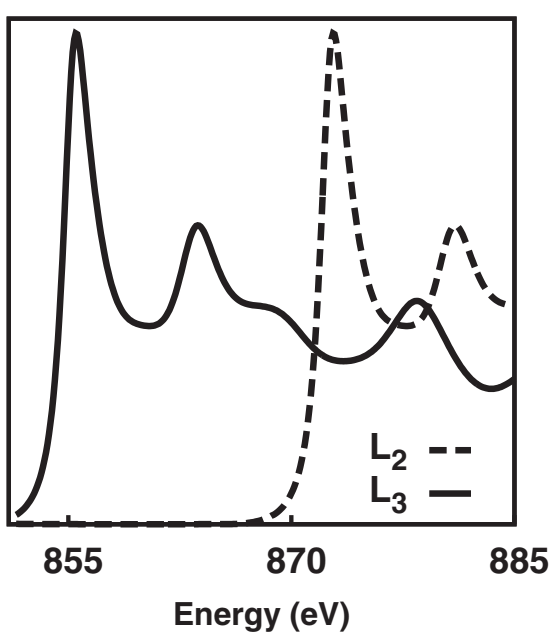

FIG. 1. Calculated real and imaginary parts of $\chi_{0}$ for the $L_{2,3}$ edges of bulk nickel. $\operatorname{Re} \chi_{0}$ is similar to $f^{\prime}$, whereas $\operatorname{Im} \chi_{0}$ indicates the absorption peaks before the multiplication with the manifold of the corresponding initial states.

the branching ratio and the position of peaks are modified. In the following we shall discuss several approximations for the exchange-correlation part of the kernel $f_{x c}$, and the physics they imply.

(f) Local-fields effect. The local fields (LFs) are due to the internal polarization of the sample when penetrated by the $\mathrm{x}$ rays. When subjected to the electromagnetic field, the electrons in the sample rearrange. The local-fields picture is coherent with the concept of particles and holes: the particle-hole pairs create an internal electric field opposed to the one of the electromagnetic wave. Indeed, one can see from Eq. (18) that the Hartree contribution is synonymous to the exchange Coulomb interaction of the particle-hole pairs. Furthermore, this identity is clearly expressed in the Bethe-Salpeter formalism. The expression in Eq. (18) is the generalization of the Slater exchange integral $G$ in the atomic multiplet theory. ${ }^{31}$ Due to the solid-state environment, the final-state wave functions
$\Psi$ contain other spherical harmonics besides the $l=2$ term, as in standard Slater integrals. Quantitatively, we found that the solid-state $1 \neq 2$ contributions are negligible.

Local fields are responsible for the screening of the x-ray field. The RPA-LF approximation to the TDDFT consists in imposing $f_{x c}^{\sigma \sigma^{\prime}}\left(\vec{r}, \vec{r}^{\prime}, \omega\right)=0$. In the language of many-body physics this is the random-phase approximation (RPA), as one only considers the exchange (no correlations) between the particle-hole pairs. Note that TDDFT in the RPA-LF limit is equivalent to the multichannel result for an unscreened core hole potential. ${ }^{13,14}$

(g) Adiabatic local-density approximation. A straightforward way to add some core-hole contribution to the RPA-LF description is to adapt the LSDA to the time-dependent situation. This method is known under the name of adiabatic local-density approximation (ALDA) or the time-dependent local (spin)-density approximation (TDLSDA). The adiabatic approximation excludes the memory effects, and the exchangecorrelation kernel depends uniquely on the density in the ground state (i.e., at $t=0$ ):

$$
f_{x c}^{\sigma \sigma^{\prime}}\left(\vec{r} t, \vec{r}^{\prime} t^{\prime}\right)=\left.\frac{\delta v_{x c}[n(t), \sigma](\vec{r})}{\delta n_{\sigma^{\prime}}\left(\vec{r}^{\prime}, t^{\prime}\right)}\right|_{n(t=0)},
$$

where $v_{x c}[n]$ is provided in Refs. 32 and 33. One sees that $f_{x c}^{\sigma \sigma^{\prime}}$ generates a potential that is local both in space and in time, and may mix the spins channels. In extended systems, the bothersome feature of the TDLSDA exchange-correlation kernel is its inaccurate long-range behavior, a consequence of the dependence on the local density. ${ }^{24}$ Moreover, TDLSDA cannot predict bound excitons, ${ }^{24}$ thus it fails to describe the core hole effects, as will be discussed in the results section. We stress the attention upon the fact that, with a view toward accuracy, we implemented the TDLSDA, and not the TDLDA, kernel. The exchange-correlation potential given by the TDLSDA in the spin unpolarized case is equal to the TDLDA one, whereas this is no longer the case of the exchange-correlation kernels, due to the mixed derivative with respect to spin in Eq. (22).

The Hartree kernel bears a positive sign, as it comes from the repulsive electron-electron interaction. On the other hand, the exchange-correlation kernel $f_{x c}$ has a negative sign which points to the core hole interaction (attractive). Consequently, local fields change the spectral weight toward higher energies, whereas the effect of the exchange-correlation kernel is opposite.

(h) Restricted adiabatic approximation. The restricted TDL(S)DA is more or less an interpolation between the RPA-LF and the TDL(S)DA. The idea is to use the TDLSDA correlation for the kernel elements that connect identical states, and the RPA-LF for the rest:

$$
\left.K^{\sigma \sigma^{\prime}}\left(\vec{r}, \vec{r}^{\prime}, \omega\right)\right|_{g g^{\prime}}=\frac{1}{\left|\vec{r}-\vec{r}^{\prime}\right|}+\delta_{g g^{\prime}} f_{x c}^{\sigma \sigma^{\prime}}(\vec{r}) \delta\left(\vec{r}-\vec{r}^{\prime}\right) .
$$

This kernel was first introduced by Ankudinov, Nesvizhskii, and $\operatorname{Rehr}^{19}$ and is supposed to provide a better description of the core hole. Should $f_{x c}$ describe the core hole, the latter's localization determines the spherical symmetry of the potential. Therefore $f_{x c}$ only couples states with identical total momentum $j$. For a detailed discussion please refer to the Results section. In Ref. 19, the kernel in Eq. (23) is 
called the dynamical TDLDA. We nevertheless prefer another nomenclature, as no explicit energy dependence appears in Eq. (23).

(i) Limitations of local kernels. The poles of the response function indicate the excitation energies in the system. Generally speaking, $\chi$ has more poles than $\chi_{0}$ does. The singularities of $\chi_{0}$ are related to the single-particle excitations in the system, whereas the extra ones introduced by $\chi$ account for the multielectron excitations (double, triple, etc., or collective). In other words, the number of electron-hole pairs is generally lower than the one of spectral terms, which is a serious limitation when $d$ electrons are present in the initial state. ${ }^{11}$ This situation is particularly visible in the case of the second half of the $3 d$ series, when the electron-hole pair picture breaks down. At the present moment, there exists no ab initio method for extended systems to solve this issue.

The approximations we made for the kernel stand within the limits of the electron-hole pair picture and cannot possibly add extra peaks to the spectrum, i.e., missing in the one-body calculation, other than plasmon ones (which belong to the optical range). To see double excitations, it is mandatory to have an energy-dependent kernel. ${ }^{34}$ This limitation concerns all the present TDDFT calculations for extended systems.

None of the kernels we introduced describes the core hole explicitly. An explicit treatment of the core hole effect implies having a kernel that describes the electron-hole interaction nonlocally, at least in space coordinates (the excitonic effect). This should be possible provided one built a kernel by exploiting the BSE formalism, ${ }^{23,35,36}$ but, since it is a singleparticle-hole approach, it would not contain the multiplet splittings. Even so, the static inclusion of the core hole effect is expected to dramatically improve the $L_{2,3}$ absorption spectra of the $3 d^{0}$ and $3 d^{1}$ elements. We believe that BSE-derived kernels can be dramatically simplified in the case of x-ray absorption, where the bound exciton (the core hole is localized inside the absorbing atom) overwhelms the others. Consequently, calculation of all electron-hole pairs (as in standard BSE) may be unnecessary. The issue here is to find an orbital dependent form of the kernel which accounts for the bound exciton, which we expect to be some limit of the BSE-derived kernel. This is beyond the scope of the work presented in this paper.

The importance of the explicit time dependence of the kernel has been recently underlined in the work of Lee and co-workers, ${ }^{37}$ who clearly state that a frequency-dependent kernel (unlike the ones derived from BSE) and memory effects are essential for the description of the multiplet splitting.

(j) Spherical harmonics expansion of the kernel. We use the spherical harmonics development of the Hartree potential, ${ }^{38}$

$$
\frac{1}{\left|\vec{r}-\vec{r}^{\prime}\right|}=\sum_{\Lambda_{0}} \frac{4 \pi}{2 l_{0}+1} \frac{r_{<}^{l_{0}}}{r_{>}^{l_{0}+1}} Y_{\Lambda_{0}}^{*}(\hat{r}) Y_{\Lambda_{0}}\left(\hat{r}^{\prime}\right),
$$

where $\Lambda_{0}=\left(l_{0}, m_{0}\right), r_{<}=\min \left(r, r^{\prime}\right)$ and $r_{>}=\max \left(r, r^{\prime}\right)$. Because of its inability to treat the singular solution, this TDDFT method requires the ground-state electronic structure to be solved by using a real potential. Therefore all the radial functions are real and we drop the H.c. for the radial functions. The contributions in Eq. (19) give

$$
\tilde{K}_{g g^{\prime}, \Lambda_{s} \Lambda_{s}^{\prime}}^{\sigma \sigma^{\prime}}(\omega)={\tilde{f_{H}}}_{g g^{\prime}, \Lambda_{s} \Lambda_{s}^{\prime}}^{\sigma \sigma^{\prime}}(\omega)+\tilde{f}_{x c}^{\sigma \sigma^{\prime}, \Lambda_{s} \Lambda_{s}^{\prime}}(\omega),
$$

where, with the aid of Eq. (24),

$$
\begin{aligned}
& {\tilde{f_{H}}}_{g g^{\prime}, \Lambda_{s} \Lambda_{s}^{\prime}}^{\sigma \sigma^{\prime}}(\omega) \\
& =c_{\Lambda_{g}^{\sigma}} c_{\Lambda_{g}^{\sigma^{\prime}}} \sum_{\Lambda_{0}} \frac{4 \pi}{2 l_{0}+1} \Gamma_{\Lambda_{\sigma}^{\prime} \Lambda_{0} \Lambda_{g}^{\sigma^{\prime}}} \Gamma_{\Lambda_{\sigma} \Lambda_{0} \Lambda_{g}^{\sigma}} \\
& \times\left(\int_{0}^{R} d r r^{1-l_{0}} b_{\Lambda_{\sigma}}^{s}\left(r, \omega+E_{g}\right) b_{g}(r) \int_{0}^{r} d r^{\prime} r^{\prime 2} b_{g^{\prime}}\left(r^{\prime}\right)\right. \\
& \times b_{\Lambda_{\sigma}^{\prime}}^{s^{\prime}}\left(r^{\prime}, \omega+E_{g^{\prime}}\right) r^{\prime} l_{0}+\int_{0}^{R} d r b_{g}(r) b_{\Lambda_{\sigma}}^{s}\left(r, \omega+E_{g}\right) \\
& \left.\times \int_{r}^{R} d r^{\prime} r^{\prime 2-l_{0}} b_{g^{\prime}}\left(r^{\prime}\right) b_{\Lambda_{\sigma}^{\prime}}^{s^{\prime}}\left(r^{\prime}, \omega+E_{g^{\prime}}\right) \frac{1}{r^{\prime} l_{0}+1}\right)
\end{aligned}
$$

and

$$
\begin{aligned}
& {\tilde{f_{x c}}}_{g g^{\prime}, \Lambda_{s} \Lambda_{s}^{\prime}}^{\sigma \sigma^{\prime}}(\omega)=c_{\Lambda_{g}^{\sigma}} c_{\Lambda_{g}^{\sigma^{\prime}}} \int_{0}^{R} d r r^{2} f_{x c}^{\sigma \sigma^{\prime}}(r) \int_{0}^{R} d r r^{2} \\
& \times b_{\Lambda_{\sigma}}^{s}\left(r, \omega+E_{g}\right) b_{g}(r) b_{g^{\prime}}(r) b_{\Lambda_{\sigma}^{\prime}}^{s^{\prime}}\left(r, \omega+E_{g^{\prime}}\right) \\
& \times \sum_{\lambda} \Gamma_{\Lambda_{\sigma} \Lambda_{g}^{\sigma} \lambda} \Gamma_{\Lambda_{g}^{\sigma^{\prime}} \Lambda_{g}^{\sigma} \lambda} .
\end{aligned}
$$

The products of type

$$
\Gamma_{\Lambda_{1} \Lambda_{2} \Lambda_{3}}=\int d \hat{r} Y_{\Lambda_{1}}^{*}(\hat{r}) Y_{\Lambda_{2}}(\hat{r}) Y_{\Lambda_{3}}(\hat{r})
$$

intervening in Eq. (26) are real quantities, also known as the Gaunt coefficients. The series in Eq. (24) can be cut at a maximum value of $l_{0}=l_{g}+l_{\max }$, according to the selection rules for the Gaunt coefficients. Furthermore, the kernels (26) and (27) prevent the mixing of odd and even states in terms of $l$. In particular this means that for $L_{2,3}$ edges $1=1(p)$ levels do not contribute to the kernel. The expression in Eq. (27) is valid for the TDLSDA, whereas the restricted scheme involves an extra $\delta_{g g^{\prime}}$ factor. The kernel expressions in both Eqs. (27) and (26) are Hermitian.

(k) Zero spin-orbit coupling limit. In the limit of no spin-orbit coupling, the solution $s$ becomes redundant. ${ }^{26}$ In this case, the solution can be identified to the spin function. All the equations presented in this paper may be simplified accordingly. In particular, the equivalence in Eq. (13) becomes

$$
\tilde{\chi}_{0 g, l m, l^{\prime} m^{\prime}}^{\sigma \sigma \prime} \equiv \tau_{l m, l^{\prime} m^{\prime}}^{\sigma \sigma \prime}
$$

\section{IMPLEMENTATION}

Any TDDFT calculation relies on a ground-state one. Consequently, the implementation of the TDDFT method within the FDMNES code is modular. From the user's point of view, it is a completely separate module, whose call is optional (via a keyword). In our implementation, the TDDFT calculation accompanies, but does not alter, the one-particle calculation. Besides the usual output files, an extra one containing the TDDFT-corrected absorption cross section is generated.

The underlying ground-state calculation, either selfconsistent or not, is equally meant to provide us the Fermi level, whose knowledge is essential for the calculation of $\chi_{0}$. The ground-state calculation can be performed either in the 
MST or in the finite difference method. ${ }^{1,39}$ The latter is to be preferred if the muffin-tin approximation is questionable for the calculated compound. ${ }^{39}$

To evaluate $\chi_{0}(\omega)$ with the aid of Eq. (14) we will be using $\operatorname{Im} \tau_{\Lambda_{s} \Lambda_{s}^{\prime}}(E)$ and $Z_{g^{\prime} \Lambda_{\sigma}^{\prime}}^{s^{\prime}}(E) Z_{g \Lambda_{\sigma}}^{s}(E)$ from the one-particle calculation. The energy dependence of $\operatorname{Im} \tau$ points out to some photoelectron energy $E$, whereas $\chi_{0}$ depends on the photons' energy $\omega$. Thus, for a TDDFT calculation where two edges are involved, it is mandatory to construct an extended energy grid that will explicitly contain the two edges. This new grid is built automatically from the initial ones, which were user defined. We superpose the two initial grids, which we previously shifted by a quantity corresponding to the energy difference between the two edges.

To get the values of $\chi_{0}$ on the extended grid, according to Eq. (14), we need to evaluate integrals of the following kind:

$$
B(\omega)=\int_{E_{F}}^{\infty} d E \frac{A(E)}{\omega-\left(E-E_{g}\right)+i \epsilon} .
$$

In practice, the upper limit of the integral is taken some $1000 \mathrm{eV}$ above the last point in the energy grid, instead of $\infty$. The missing values in $A(E)$ are taken as the extrapolation of the imaginary part of the atomic structure factor $f^{\prime \prime}$, with the corresponding units. The physical reason one needs a large extension of the grid is that, although $\operatorname{Im} A(E)$ are very localized structures around the edge energies, $\operatorname{Re} A(E)$ are extended. Note that this procedure of calculating the real part of the susceptibility is proper to our implementation. The other existing methods (see the implementation details in Refs. 17 and 19) use the Kramers-Kronig relations.

One can evaluate Eq. (30) either by taking the limit $\epsilon \rightarrow 0$ or by applying a finite broadening. In practice, applying a broadening in the very form of $\chi_{0}$ or only at the end of the absorption calculation give similar results. The former is necessary if one chooses distinct convolution widths at the two edges, in which case the latter procedure cannot be used. Note that we obtain a $\chi_{0}$ representation of each edge $g$, whose imaginary parts' unique peaks are similar in form but shifted in energy (see Fig. 1). The two different peaks have identical heights, as the difference in the spectral weight of the absorption peaks is due to the sum on the initial states, a later step in the calculation.

The main idea behind this particular TDDFT method is that we can treat $\tilde{\chi}$ and $\tau$ on equal footing. Once we get $\tilde{\chi}$ we reuse the one-particle implementation in the code that was meant for $\tau$ and calculate the tensors in the usual FDMNES way. This procedure is extremely convenient, as we get to "recycle" the ancient structure of FDMNES and keep the powerful feature of the tensor analysis.

One great advantage of the TDDFT calculation is that it is not time consuming. Most of the computational time goes into the matrix inversion in Eq. (21). A remarkable fact is that the TDDFT calculation time does not scale with the radius of the cluster. Due to the approximation for resonant regimes in the Adler-Wiser equation (7), $\chi_{0}$ only takes significant values inside the absorbing atom. This means that the matrix inversion in Eq. (21) is limited to the spacial range of the absorber, and thus the computation time required for the TDDFT method is nearly independent of the size of the calculation cluster.
In principle, our TDDFT scheme is able to calculate the absorption cross section (1) beyond the electric dipole approximation. Indeed, should one consider the electric quadrupole contribution to the field operator $\hat{O}$ in Eq. (12) and given the equivalence in Eq. (13), the response function will contain the quadrupolar term.

\section{RESULTS}

To test the pertinence of our method, we calculated the TDDFT corrections for the $L_{2,3}$ absorption spectra of the bulk transitional elements. We used large clusters of calculation (7 $\AA$ ) and the cutoff level issued from our self-consistent procedure. The experimental data issue from Refs. 9 and 40. The former is used as reference by Schwitalla and Ebert ${ }^{17}$ and Ankudinov, Nesvizhskii, and Rehr. ${ }^{19}$ Whenever available, we prefer the more recent data in Ref. 40, for the reason that it has better experimental resolution.

In our series of calculations, we included calcium and scandium, due to their available $3 d$ states, and excluded zinc, whose $3 d$ levels are full. For all these elements we compared the single-particle calculations (LSDA) to the TDDFT ones (RPA-LF, TDLSDA, restricted TDLSDA) for identical broadening. In order not to artificially alter the branching ratio we chose a broadening that does not depend on the energy. We calculate the branching ratio by a simple ratio of the maxima of the intensities of the $L_{3}$ and $L_{2}$ structures, in a similar way as in Refs. 17 and 19. The TDDFT results we show are issued from fully relativistic calculations preceded by a ground-state self-consistent loop. ${ }^{41}$ All calculations shown for the magnetic $3 d$ elements (iron, cobalt, nickel) include the spin polarization.

In Figs. 2-4 we compare LSDA and TDLSDA calculations to experimental data, for the $L_{2,3}$ edges of the $3 d$ elements. TDDFT definitely improves on the one-body calculations, for the first half of the $3 d$ series, both in terms of branching ratio and general shape of the spectra. The experimental data were normalized to match the high-energy spectrum of the calculations. Neither the LSDA nor the TDLSDA calculations were normalized, which allows one to observe the eventual energy shift and the mismatch at high energies. The TDDFT corrections have little influence for the second half of the $3 d$ series, and are sometimes worse than the LSDA ones (see the case of iron and cobalt in Fig. 4).

As a general remark, the TDDFT and the one-body calculations meet at high energies. When this superposition is not perfect (see the case of calcium, scandium, and nickel in Figs. 2 and 4) we suspect the inappropriateness of the local kernel applied to that particular case. Indeed, the calculated spectral shape for these materials is not satisfactory, although in the case of calcium and scandium the branching ratio is improved by the TDDFT calculation. One expects that the many-body effects are limited to low energies. Such behavior is confirmed by the multichannel theory: ${ }^{12}$ the adiabatic limit leads to the sudden approximation at high energies.

(l) Various local kernels. One can see (Figs. 5 and 6) that the various exchange-correlation kernels that we tested (RPA-LF, TDLSDA, restricted TDLSDA) give quasi-identical results. This result is in agreement with the findings of Schwitalla and Ebert $^{17}$ and turns to be valid equally for TDDFT calculations 

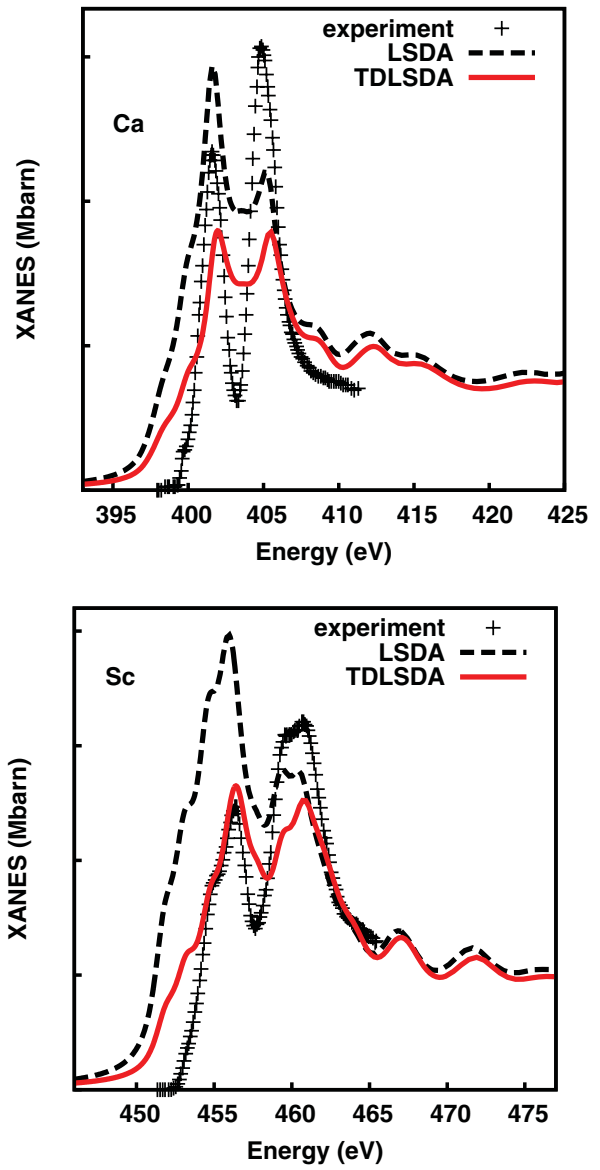

FIG. 2. (Color online) One-body LSDA (dashes) and TDLSDA calculations (solid) versus experiment (Ref. 9) (dots) for calcium and scandium at the $L_{2,3}$ edges. TDLSDA improves on the LSDA calculations, but not enough to give a satisfactory agreement with the experiment in terms of general shape of the spectra. Both calculations seem to underestimate the onset of the $L_{3}$ edge.

on complex structures (for instance, transition elements oxides, not shown here). From the physics point of view, this means that at the $L_{2,3}$ edges of $3 d$ elements the local-fields effect is important, whereas the local part of the electron-hole interaction is negligible. For $K$ edges (not shown here) we found that both local fields and LDA exchange correlation are negligible, i.e., TDDFT results are identical to DFT ones. We understand this as an effect of the reduced overlap between the core $1 s$ state and the empty $p$ levels. Furthermore, in TDL(S)DA, to have an effect, we need to mix transitions belonging to different edges (we checked that interedge mixing, as occurring at $K$ edges, is indeed negligible). Hence inclusion of core hole effects (i.e., a nonlocal TDDFT kernel) is mandatory for improving the description of $K$ edges. We draw attention to the fact that the quantitative insignificance of $f_{x c}$ is essentially different different from the optical frequency range, where the inclusion of the local exchange-correlation kernel generates an effect that is visible on the spectra. It appears that in the $\mathrm{x}$-ray range the interaction with the core hole has an extremely pronounced nonlocal character. Consequently, we argue that the use of a nonlocal exchange-correlation kernel is mandatory for accurate TDDFT $\mathrm{x}$-ray absorption calculations.
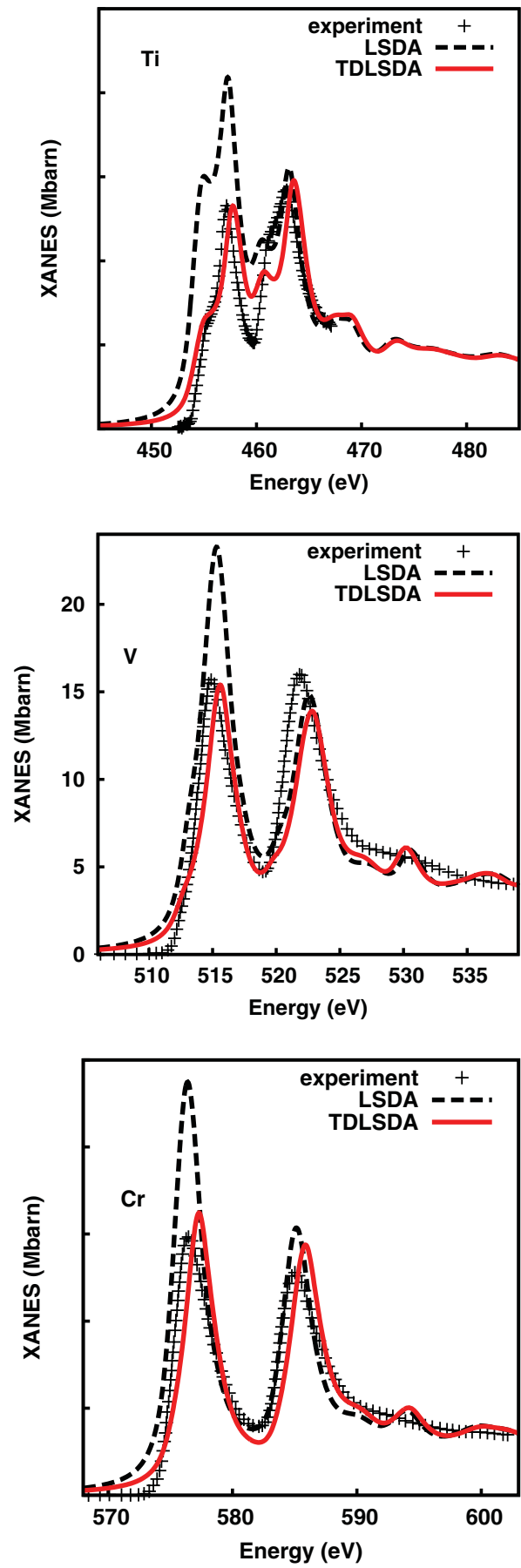

FIG. 3. (Color online) One-body LSDA (dashes) and TDLSDA calculations (solid) versus experiment (Ref. 40) (dots) for titanium, vanadium, and chromium at the $L_{2,3}$ edges. Among the $3 d$ transition metals, for these elements the agreement of the TDDFT calculation and experimental data is the best one.

For the first half of the $3 d$ series our results are in good agreement with the ones of Schwitalla and Ebert. ${ }^{17}$ They obtain a TDDFT correction that reduces slightly with the increasing atomic number. In our case, this tendency is stronger and leads to an almost zero effect in the case of nickel. Our results can be understood as follows. The quantitative change in the branching ratio within TDLSDA is directly proportional to the inverse of the $2 p$ spin-orbit coupling and to 

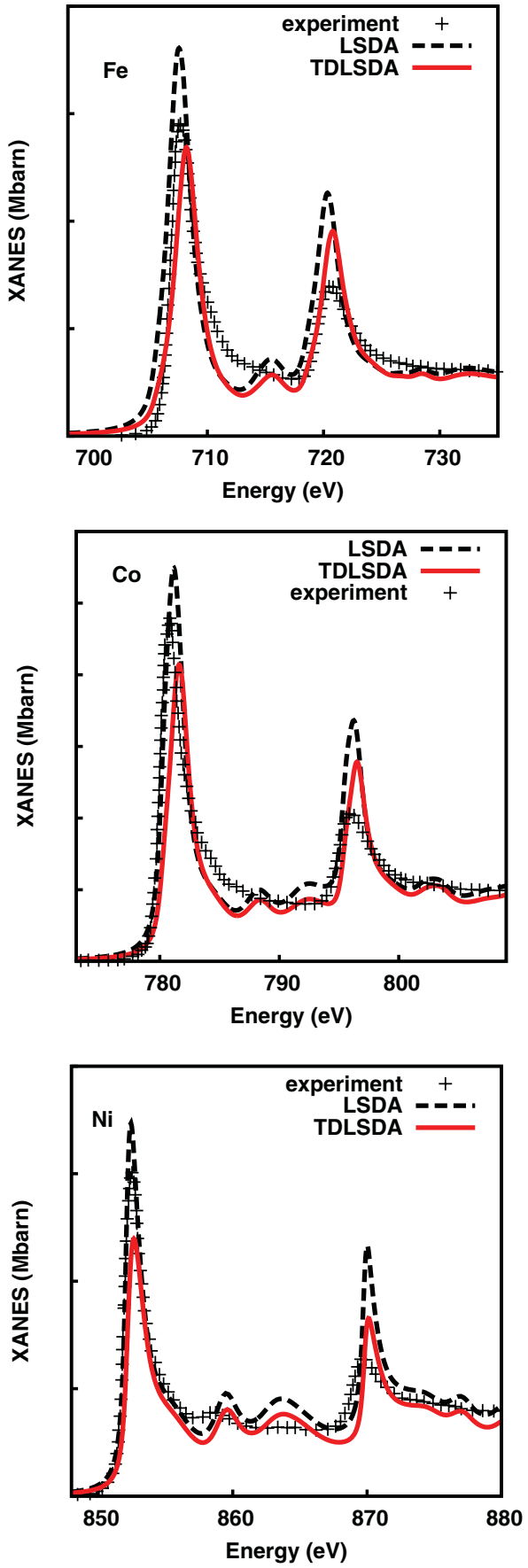

FIG. 4. (Color online) One-body LSDA (dashes) and TDLSDA calculations (solid) versus experiment (Ref. 40) for iron, cobalt, and nickel at the $L_{2,3}$ edges. Insofar as the branching ration is concerned, the TDLSDA corrections are negligible for nickel, and worsen the LSDA results for iron and cobalt.

the generalized Slater exchange integral. ${ }^{13}$ They both decrease with the increasing of the atomic number in the $3 d$ series. In particular, the atomic Slater exchange completely cancels for the $3 d^{9}$ configuration, ${ }^{31}$ thus the TDLSDA calculations on nickel $\left(3 d^{8}\right)$ should be close, if not identical, to the LSDA ones. Generally speaking, TDDFT is a particle-hole theory and expresses a particular excitation as the linear combination of single-particle-hole pairs. However, the single excitation

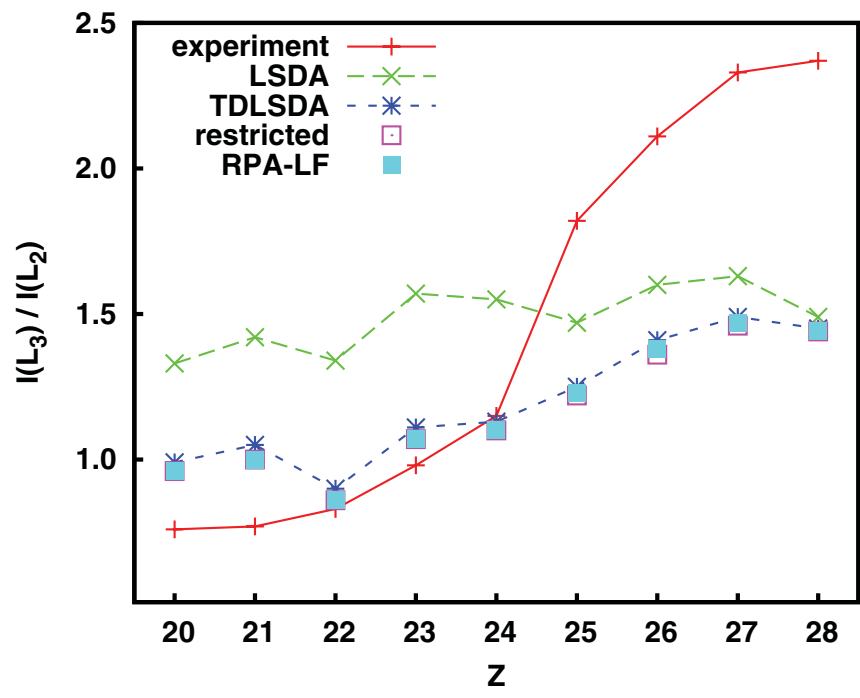

FIG. 5. (Color online) The $L_{2,3}$ branching ratios: the TDDFT corrections improve the LSDA calculations for the first elements of the $3 d$ series. The different local exchange-correlation kernels (RPA-LF, TDLSDA, and restricted TDLSDA) give quasi-identical results. This figure has been made with the data in Table I.

picture breaks down for the late $3 d$ elements, because of the presence of $d$ electrons in the ground state. ${ }^{11}$

Our results disagree with the ones of Refs. 19 and 42 upon the importance of the local exchange-correlation effects. These authors find a large difference between the branching ratio calculated in RPA-LF with respect to the TDLSDA one. They equally find a good match between the restricted TDLSDA calculated and the experimental branching ratio at the $L_{2,3}$

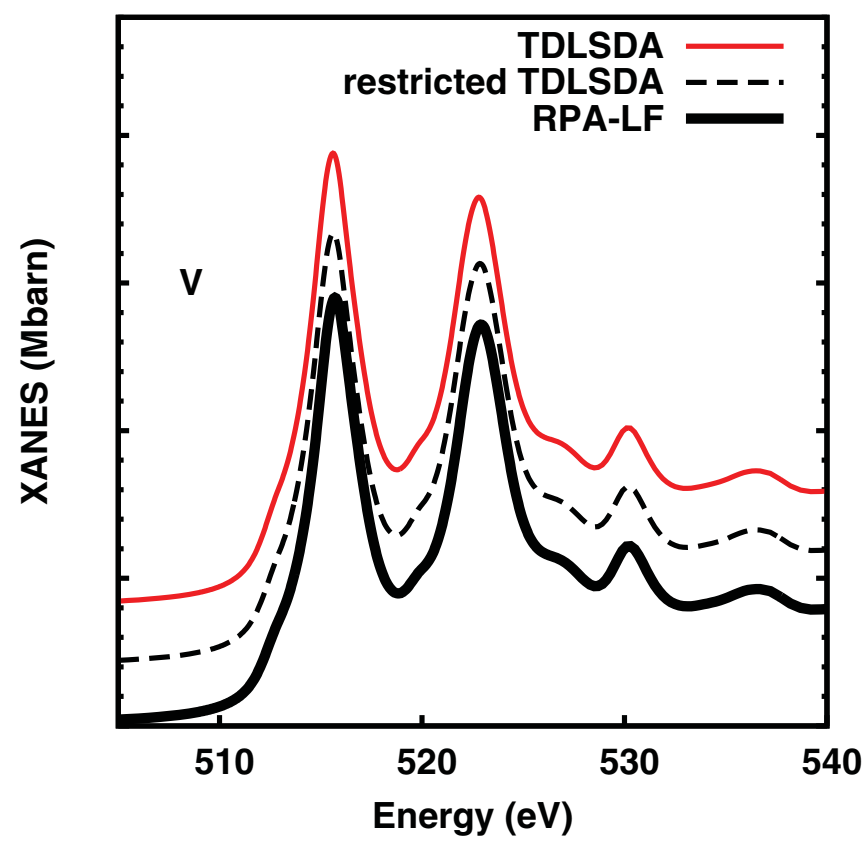

FIG. 6. (Color online) TDDFT calculations of the $L_{2,3}$ edges of vanadium. Three distinct local approximations for the exchangecorrelation kernel have been tested: RPA-LF (thick solid), TDLSDA (thin solid), and restricted TDLSDA (dashes). Calculations were shifted vertically for visibility. They all yield similar results. 
TABLE I. The $L_{2,3}$ branching ratio, experimental and calculated. SO stands for spin-orbit calculation.

\begin{tabular}{|c|c|c|c|c|c|c|}
\hline \multirow[b]{2}{*}{ Method } & \multicolumn{2}{|c|}{$\mathrm{Ca}$} & \multicolumn{2}{|c|}{$\mathrm{Sc}$} & \multicolumn{2}{|c|}{$\mathrm{Ti}$} \\
\hline & $\mathrm{SO}$ & no SO & $\mathrm{SO}$ & no SO & SO & no SO \\
\hline LSDA & 1.33 & 1.31 & 1.42 & 1.42 & 1.34 & 1.34 \\
\hline RPA-LF & 0.96 & 1.16 & 1.00 & 1.21 & 0.86 & 0.72 \\
\hline TDLSDA & 0.99 & 1.23 & 1.05 & 1.30 & 0.90 & 0.83 \\
\hline Restricted TDLSDA & 0.96 & 1.17 & 1.00 & 1.25 & 0.85 & 0.76 \\
\hline \multirow[t]{2}{*}{ Experiment } & \multicolumn{2}{|c|}{$0.76^{\mathrm{a}}$} & \multicolumn{2}{|c|}{$0.77^{\mathrm{b}}$} & \multicolumn{2}{|c|}{$0.83^{b}$} \\
\hline & \multicolumn{2}{|c|}{$\mathrm{V}$} & \multicolumn{2}{|c|}{$\mathrm{Cr}$} & \multicolumn{2}{|c|}{$\mathrm{Mn}$} \\
\hline Method & $\mathrm{SO}$ & no SO & $\mathrm{SO}$ & no SO & $\mathrm{SO}$ & no SO \\
\hline$\overline{\text { LSDA }}$ & 1.57 & 1.58 & 1.55 & 1.55 & 1.64 & 1.55 \\
\hline RPA-LF & 1.07 & 1.26 & 1.10 & 1.21 & 1.24 & 1.29 \\
\hline TDLSDA & 1.11 & 1.29 & 1.13 & 1.22 & 1.26 & 1.32 \\
\hline Restricted TDLSDA & 1.07 & 1.25 & 1.10 & 1.19 & 1.25 & 1.30 \\
\hline \multirow{2}{*}{ Experiment } & \multicolumn{2}{|c|}{$0.98^{b}$} & \multicolumn{2}{|c|}{$1.15^{\mathrm{b}}$} & \multicolumn{2}{|c|}{$1.82^{\mathrm{a}}$} \\
\hline & \multicolumn{2}{|c|}{$\mathrm{Fe}$} & \multicolumn{2}{|c|}{ Co } & \multicolumn{2}{|c|}{$\mathrm{Ni}$} \\
\hline Method & $\mathrm{SO}$ & no SO & $\mathrm{SO}$ & no SO & $\mathrm{SO}$ & no SO \\
\hline LSDA & 1.60 & 1.76 & 1.63 & 1.71 & 1.49 & 1.39 \\
\hline RPA-LF & 1.38 & 1.40 & 1.47 & 1.49 & 1.44 & 1.32 \\
\hline TDLSDA & 1.41 & 1.39 & 1.49 & 1.43 & 1.45 & 1.35 \\
\hline Restricted TDLSDA & 1.36 & 1.38 & 1.46 & 1.44 & 1.44 & 1.34 \\
\hline Experiment & \multicolumn{2}{|c|}{$2.11^{\mathrm{b}}$} & \multicolumn{2}{|c|}{$2.33^{\mathrm{b}}$} & \multicolumn{2}{|c|}{$2.37^{\mathrm{b}}$} \\
\hline
\end{tabular}

assue from experimental data taken from Ref. 9.

${ }^{\mathrm{b}}$ Issue from experimental data taken from Ref. 40.

branching ratio for the late $3 d$ elements. Our calculations lead us to different conclusions, as explained above.

(m) Relativistic effects. We performed two different sets of calculations, with and without the spin-orbit coupling on the $3 d$ states (see Tables I and II). We found an intrinsic effect within TDDFT, due to relativity. In this work, when we contrast relativistic and nonrelativistic calculations we refer to considering or not the spin-orbit coupling effect on the $d$ states (for the $2 p$ it is considered anyway). Hence relativistic TDDFT means that we use a four quantum number basis for the expansion of the kernel and response functions in Eqs. (10), (11), and (18). Even if for the first elements of the series the spin-orbit coupling makes no difference in terms of the one body calculations, as the orbital moment is quenched, this is no longer the case for the TDDFT ones (see Fig. 7). The difference comes from the crossed spin RPA-LF kernel matrix elements. This is intrinsically relativistic, in the sense that even for zero $3 d$ spin-orbit coupling the contribution of $\chi_{\uparrow \downarrow}$ to the absorption cross section is significant. Should one perform scalar TDDFT, the result is similar to the one obtained by retaining only the RPA-LF kernel elements diagonal in spin (Fig. 7). Hence scalar TDDFT erases most of the effect of the spin crossing. The importance of the latter has equally been detected in multichannel calculations. ${ }^{43}$ Therefore TDDFT calculations should be performed in the fully relativistic framework for all

TABLE II. The values (in eV) of the convolution parameter $\Gamma$ one has used to calculate the branching ratio in Table I.

\begin{tabular}{lllllllll}
\hline \hline $\mathrm{Ca}$ & $\mathrm{Sc}$ & $\mathrm{Ti}$ & $\mathrm{V}$ & $\mathrm{Cr}$ & $\mathrm{Mn}$ & $\mathrm{Fe}$ & $\mathrm{Co}$ & $\mathrm{Ni}$ \\
\hline 1.29 & 1.36 & 1.43 & 1.50 & 1.56 & 1.63 & 0.60 & 0.80 & 0.60 \\
\hline
\end{tabular}

materials, regardless of the atomic weight or spin polarization. This is a remarkable effect and, to our knowledge, it is for the first time it has been seen (for core excitations, at least). Note that in this sense our implementation is equivalent to the one in Ref. 17 but, to our understanding, is more powerful than the

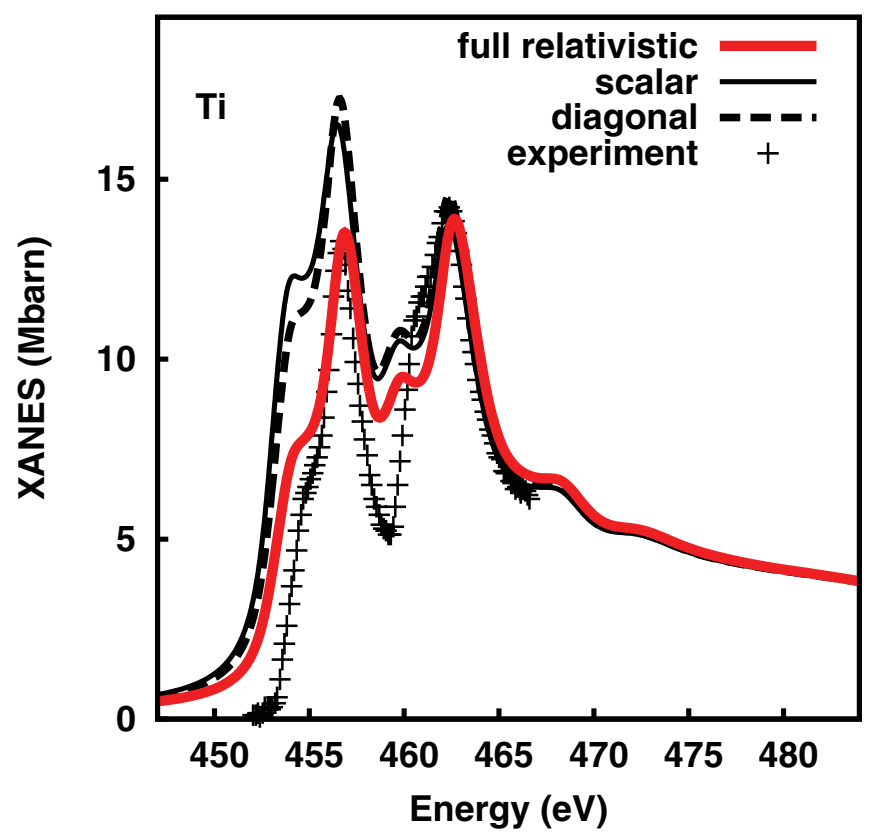

FIG. 7. (Color online) TDDFT calculations of the $L_{2,3}$ edges of titanium: scalar (thin solid) and relativistic (thick solid). The difference between the two spectra seems to come mainly from the spin nondiagonal Coulomb kernel matrix elements (calculation in dashes). Experimental data (dots) were taken from Ref. 9. 
scalar one introduced in Ref. 19. In practice, to save calculation time, whenever we deal with $3 d$ elements, we perform a scalar (no spin-orbit coupling) ground-state calculation that we inject into the relativistic TDDFT calculation. We checked, analytically and numerically, that we get the same results as if we had used a relativistic ground state as a prior step to the TDDFT procedure.

(n) Mixing of representations. In the one-body approximation $\chi$ may not couple two $\Lambda$ states that belong to distinct point-group representations. Once the mixing of transitions is tuned on, this statement is no longer valid. For instance, for octahedral compounds, the structures in the one-body spectrum are assigned to the $t_{2 g}$ and $e_{g}$ orbitals in the density of states. Should one describe some many-body effects like the local fields, one no longer measures the density of states. In this sense, one could understand that the spectral structures correspond to a mixing of the $e_{g}$ and $t_{2 g}$ character. This does not mean that RPA-LF lowers the point symmetry around the absorbing atom, but that the particle-hole channel mixing induces a similar superposition of the final states.

As a quantitative example we refer to the titanium $L_{2,3}$ edges in $\mathrm{FeTiO}_{3}$. The titanium's environment is a distorted octahedron whose point group is $C_{3}$. Therefore, in terms of complex spherical harmonics, the $d$ orbitals belong either to the $\mathcal{E}(\mathrm{m}= \pm 1, \pm 2)$ or to the totally symmetric representation $\mathcal{A}(\mathrm{m}=0)$. In Fig. 8 we show the contribution to the RPA-LF spectra of each irreducible representation. Note that contrary to the one-body approximation, the total absorption spectrum issued from the TDDFT calculation is different from the sum of $\mathcal{A}-\mathcal{A}$ and $\mathcal{E}-\mathcal{E}$ contribution. Consequently, there is a nonvanishing contribution corresponding to the $\mathcal{A}-\mathcal{E}$ and $\mathcal{E}-\mathcal{A}$ cross terms. As expected, the sum of all contributions yields the total RPA-LF signal. Note that in this calculation only the $d$ orbitals are of interest. Indeed, we found that the $s(l=0)$ RPA-LF kernel contribution to $\mathcal{A}$ is negligible, whereas the $p$ $(l=1)$ terms are forbidden by the selection rule on the kernel elements, as stated previously.

The mixing of representations is stronger in the fully relativistic case (the upper panel of Fig. 8) than in the scalar one (middle panel), due to the $\chi_{\uparrow \downarrow}$ contribution that is ignored by the latter. Once again, one can see the necessity of performing fully relativistic TDDFT calculations. Indeed, the correct estimation of the cross term leads to a satisfactory agreement with the experiment ${ }^{44}$ (the lower panel of Fig. 8). All calculations were performed on clusters containing 66 atoms and the convolution widths used are 0.5 and $1 \mathrm{eV}$, at the $L_{3}$ and $L_{2}$ edge, respectively.

(o) TDDFT on excited atoms. All the TDDFT calculations described above have been performed on an underlying ground-state calculation. We also tried to insert the final-state rule in this preliminary calculation. We described a cluster with a fully screened core hole, and calculated $\hat{\chi}_{0}$ accordingly. Quantitatively, this procedure turned up to worsen the agreement with the experiment, meaning that a fully screened core is a bad ground-state solution for our TDDFT calculation. Consequently we chose not to pursue this direction. Such a procedure is not rigorously justified, as the Adler-Wiser equations (7) require the wave functions $\Psi_{\Lambda}$ to be calculated in the ground state. Nevertheless, it is known that in solid state the core hole is strongly screened. ${ }^{11}$ We therefore tried
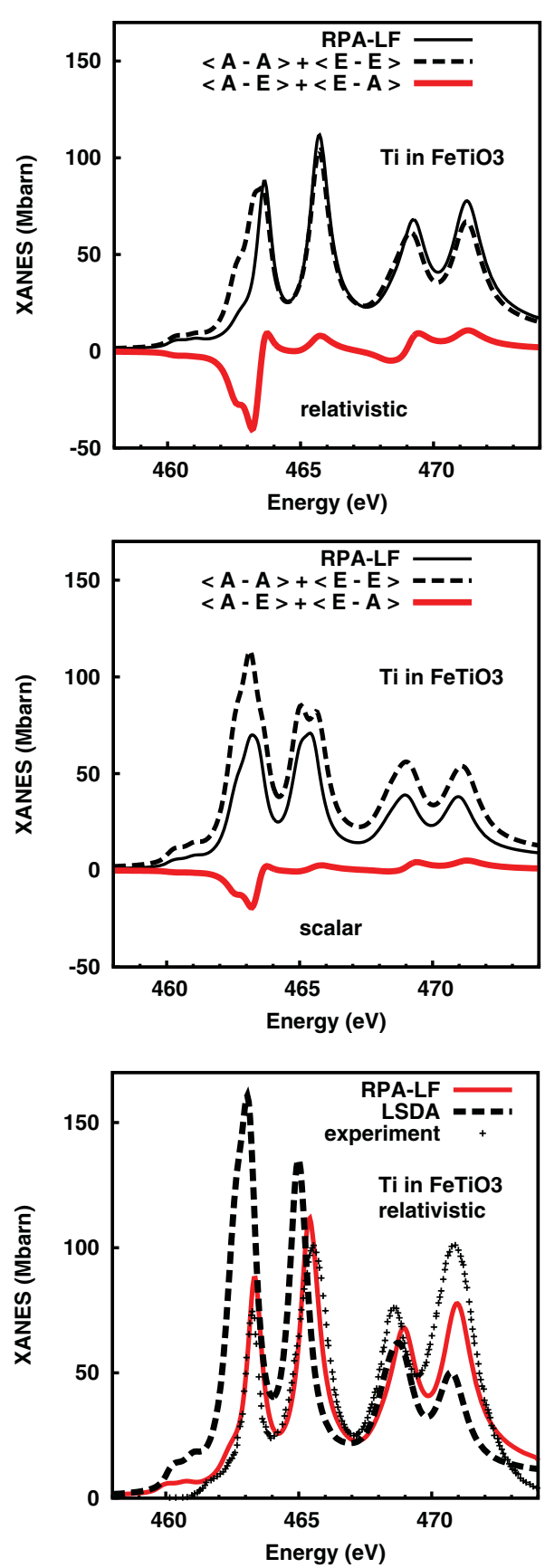

FIG. 8. (Color online) Symmetry analysis of the RPA-LF calculation at the titanium $L_{2,3}$ edges in $\mathrm{FeTiO}_{3}$. The $d$ states belong to either the $\mathcal{A}$ or $\mathcal{E}$ irreducible representations. The core hole causes the emergence of a $\mathcal{A}-\mathcal{E}$ cross term (solid line), which is absent in the ground-state calculation. This effect is underestimated by the scalar calculation (middle panel). Once again, relativistic effects are crucial in order to obtain the quantitative estimation of the cross terms as well as a satisfactory agreement with the experimental data (Ref. 44). In the lower panel we confront the LSDA calculations (dashes) and the fully relativistic RPA-LF ones (solid) to the experimental data (dots).

to take into account this effect by performing a self-consistent calculation with the core hole. This issue remains open.

(p) Cluster size. We checked that our TDDFT procedure had no intrinsic artifacts due to the size of the calculation cluster, provided it contained at least one atomic shell around the absorbing atom. As we explained previously, the effect of 


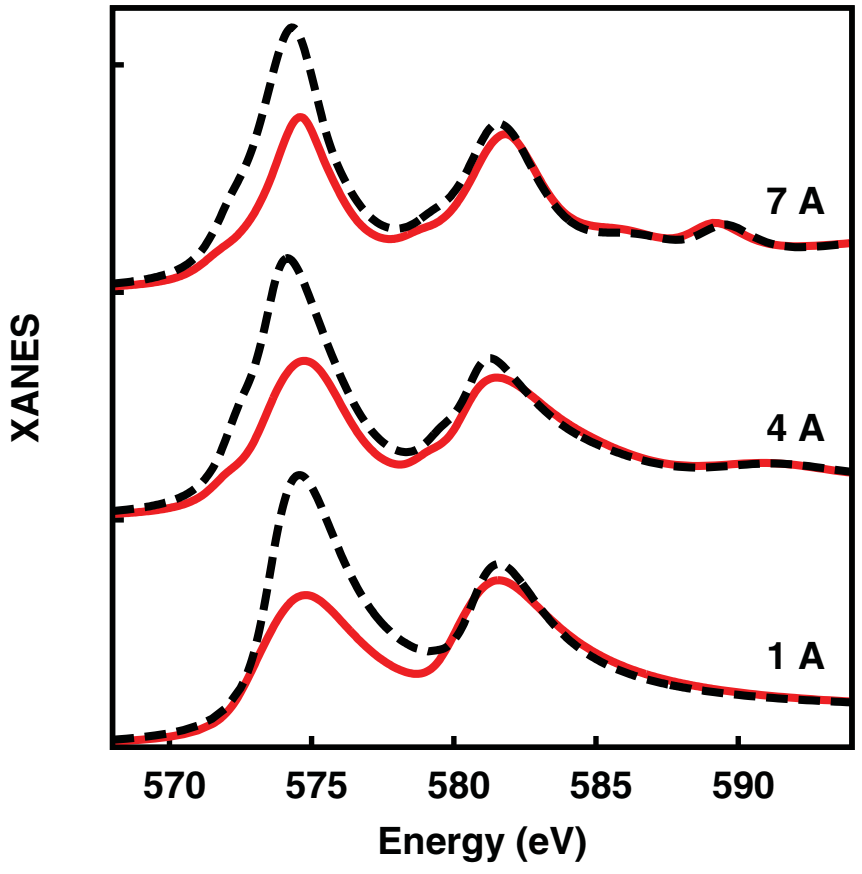

FIG. 9. (Color online) The absorption at the $L_{2,3}$ edges of vanadium, for different sizes of the calculation cluster: $1 \AA$ (a single atom), $4 \AA$ (15 atoms), and $7 \AA$ (113 atoms). The TDDFT spectra (solid) are always beneath the corresponding one-body ones (dashes). Spectra corresponding to different radii are vertically shifted for convenience.

the TDDFT is localized around the absorbing atom. Therefore, when increasing the cluster's radius, the form of the TDDFT spectrum follows accurately the band modulations of the LSDA one (see Fig. 9). It follows that most of the change brought in by the TDDFT is independent of the size of the cluster. The difference between the LSDA and TDDFT calculations of absorption in vanadium is roughly the same, whether one deals with a single atom or with a cluster.

(q) TDDFT and the mixing of transition channels. We found that performing a TDDFT calculation with a local kernel, at a single edge, as the $K$ edge of the $3 d$ elements, yields practically the same values as its LSDA correspondent. It follows that TDLSDA can only account for the local fields or exchange-correlation effects through the mechanism of the mixing of transitions, which occurs when one solves (in a single calculation) some adjacent edges, like the $L_{2,3}$ edges of the $3 d$ elements. Although in principle the TDDFT correction should also affect the isolated edges (for instance, the $K$-edge prepeaks), we never managed to have a quantitative effect. We believe this is a consequence of the local approximation on the exchange-correlation kernel, as explained in a previous paragraph.

\section{CONCLUSIONS}

We presented a detailed study of adiabatic TDDFT methods applied to X-ray absorption at the $L_{2,3}$ edges. We have shown that the TDDFT with local kernels improves on the LSDA calculations for the first half of the $3 d$ series, and especially for titanium, vanadium, and chromium. This is no longer the case for the second half, indicating that an essential ingredient is still missing from our TDDFT scheme. We believe that same as for the optical region, at X-ray frequencies the inclusion of the core hole is essential in order to get a satisfactory agreement with the experimental data. It has been argued ${ }^{23}$ that the TDLSDA should give satisfactory results for core spectroscopy. Our results invalidate this statement, therefore a nonlocal (and eventually frequency-dependent) exchange-correlation kernel is necessary for the TDDFT calculation of core spectroscopy.

Our conclusion is in reasonable agreement with the work of Schwitalla and Ebert ${ }^{17}$ and Ankudinov, Nesvizhskii, and Rehr ${ }^{19}$ on local-fields effects. On the other hand, some of our results contradict the findings of the latter, ${ }^{19}$ who achieved a good agreement with the experimental values of the $L_{2,3}$ branching ratios for the late $3 d$ elements, all by employing a local kernel (the restricted TDLSDA). Contrary to the conclusion in Ref. 19, our calculations indicate that all the local exchange-correlation kernels we employed (RPA-LF, TDLSDA, restricted TDLSDA) yield similar results.

To our knowledge, it is the first time that the importance of the fully relativistic TDDFT treatment of $3 d$ elements has been stated. Peculiar effects arise upon the spin crossing in the response function $\chi$, that would be disregarded in a scalar TDDFT calculation.

Our method is in principle equivalent to the TDDFT quantum chemistry calculations. ${ }^{20,45,46}$ Nonetheless, our use of the Dyson equation, as opposed to the Casida formulation in quantum chemistry, renders our calculation more appropriate to extended crystals. Indeed, quantum chemistry calculations seem limited to small cluster sizes (a few atoms) provided relativistic effects are included into the description.

There are clear shortcomings of the TDDFT calculations on extended systems, at the present moment. The most stringent ones concern the validity of TDDFT for open-shell systems, the prediction of excitonic lines and multiplet splitting, or the correct position of $E 2-E 2$ structures at $K$ edges. It remains unclear to what extent the limitations of the underlying DFTLDA ground-state calculation affect the performance of the TDDFT calculation. Insofar as the latter is concerned, the exchange-correlation kernel accounting for all the many-body effects (including multiplets) has not been derived yet.

The x-ray absorption community lacks a convenient calculation tool that is able to account for the core hole. This has been achieved by BSE calculations ${ }^{15,16}$ but at a rather high computational cost, mainly because of the use of four-point quantities. We strongly believe that TDDFT is a good candidate for such task, and considerably less computer demanding as it deals with two point quantities. One of the perspectives of this work includes the implementation of a kernel supposed to describe the excitonic effects, in the simplified situation where the core hole is localized, which is the case of x-ray absorption spectroscopy. Second, we wish to test the way in which our TDDFT procedure couples to the LSDA $+U$ ground-state calculations. The calculation of the $L_{2,3}$ edges of correlated materials is expected to benefit from this $d u o$.

\section{ACKNOWLEDGMENTS}

The authors would like to thank Peter Krüger and Calogero Natoli for fruitful discussions. 
"bunau@impmc.upmc.fr

${ }^{1}$ Y. Joly, Phys. Rev. B 63, 125120 (2001).

${ }^{2}$ C. R. Natoli, D. K. Misemer, S. Doniach, and F. W. Kutzler, Phys. Rev. A 22, 1104 (1980).

${ }^{3}$ M. Benfatto, S. Della Longa, and C. R. Natoli, J. Synchrotron Radiat. 10, 51 (2003).

${ }^{4}$ A. L. Ankudinov, B. Ravel, J. J. Rehr, and S. D. Conradson, Phys. Rev. B 58, 7565 (1998).

${ }^{5}$ D. Cabaret, E. Gaudry, M. Taillefumier, P. Sainctavit, and F. Mauri, Phys. Scr. T115, 131 (2005).

${ }^{6}$ P. Blaha, K. Schwarz, G. K. H. Madsen, D. Kvasnicka, and J. Luitz, in WIEN2k An Augmented Plane Wave Plus Local Orbital Program for Calculating Crystal Properties (TU Wien, Austria, 2001), ISBN 3-9501031-1-2.

${ }^{7}$ H. Ebert, Electronic Structure and Physical Properties of Solids (Springer, Berlin, 2000), Vol. 535, p. 191.

${ }^{8}$ F. de Groot, Coord. Chem. Rev. 249, 31 (2005).

${ }^{9}$ J. Fink, T. Müller-Heinzerling, B. Scheerer, W. Speier, F. U. Hillebrecht, J. C. Fuggle, J. Zaanen, and G. A. Sawatzky, Phys. Rev. B 32, 4899 (1985).

${ }^{10}$ A. Zangwill and P. Soven, Phys. Rev. A 21, 1561 (1980).

${ }^{11}$ J. Zaanen, G. A. Sawatzky, J. Fink, W. Speier, and J. C. Fuggle, Phys. Rev. B 32, 4905 (1985).

${ }^{12}$ C. R. Natoli, M. Benfatto, C. Brouder, M. F. Ruiz López, and D. L. Foulis, Phys. Rev. B 42, 1944 (1990).

${ }^{13}$ P. Krüger and C. R. Natoli, Phys. Rev. B 70, 245120 (2004).

${ }^{14}$ P. Krüger, Phys. Rev. B 81, 125121 (2010).

${ }^{15}$ E. L. Shirley, Phys. Rev. Lett. 80, 794 (1998).

${ }^{16}$ R. Laskowski and P. Blaha, Phys. Rev. B 82, 205104 (2010).

${ }^{17}$ J. Schwitalla and H. Ebert, Phys. Rev. Lett. 80, 4586 (1998).

${ }^{18}$ Z. Levine, J. Phys. B 31, 3155 (1998).

${ }^{19}$ A. L. Ankudinov, A. I. Nesvizhskii, and J. J. Rehr, Phys. Rev. B 67, 115120 (2003).

${ }^{20}$ M. Stener, G. Fronzoni, and M. de Simone, Chem. Phys. Lett. 373, 115 (2003).

${ }^{21}$ A. L. Ankudinov, Y. Takimoto, and J. J. Rehr, Phys. Rev. B 71, 165110 (2005).

${ }^{22}$ E. Runge and E. K. U. Gross, Phys. Rev. Lett. 52, 997 (1984).

${ }^{23}$ S. Botti, A. Schindlmayr, R. Del Sole, and L. Reining, Rep. Prog. Phys. 70, 357 (2007).
${ }^{24}$ G. Onida, L. Reining, and A. Rubio, Rev. Mod. Phys. 74, 601 (2002).

${ }^{25}$ M. Marques, U. C., F. Noguiera, A. Rubio, K. Burke, and E. Gross, Time Dependent Density Functional Theory (Springer, Berlin Heidelberg, 2006).

${ }^{26}$ Y. Joly, in Magnetism and Synchrotron Radiation, edited by E. Beaupaire, H. Bulou, F. Scheurer, and J. Kappler (Springer, Berlin Heidelberg, 2010), pp. 77-126.

${ }^{27}$ J. H. Wood and A. M. Boring, Phys. Rev. B 18, 2701 (1978).

${ }^{28}$ Y. Joly, S. Di Matteo, and C. R. Natoli, Phys. Rev. B 69, 224401 (2004).

${ }^{29}$ C. R. Natoli, D. K. Misemer, S. Doniach, and F. W. Kutzler, Phys. Rev. A 22, 1104 (1980).

${ }^{30} \mathrm{~F}$. Neese (private communication).

${ }^{31}$ E. Condon and G. H. Shortely, The Theory of Atomic Spectra (Cambridge University Press, Cambridge, United Kingdom, 1951).

${ }^{32}$ L. Hedin and B. Lundqvist, J. Phys. C 4, 2064 (1971).

${ }^{33}$ L. Hedin and U. von Barth, J. Phys. C 5, 1629 (1972).

${ }^{34}$ N. Maitra, F. Zhang, R. Cave, and K. Burke, J. Chem. Phys. 120, 1601 (2004).

${ }^{35}$ F. Sottile, V. Olevano, and L. Reining, Phys. Rev. Lett. 91, 056402 (2003).

${ }^{36}$ M. Gatti, V. Olevano, L. Reining, and I. V. Tokatly, Phys. Rev. Lett. 99, 057401 (2007).

${ }^{37}$ C.-C. Lee, H. C. Hsueh, and W. Ku, Phys. Rev. B 82, 081106 (2010).

${ }^{38}$ J. Angyan, I. Greber, and M. Marsman, J. Phys. A 39, 8613 (2006).

${ }^{39}$ O. Bunau and Y. Joly (to appear in J. Phys: Condens. Matter).

${ }^{40}$ A. Scherz, Ph.D. dissertation, Freie Universitat, Berlin, 2004 [http://www-ssrl.slac.stanford.edu/stohr].

${ }^{41}$ O. Bunău and Y. Joly, J. Phys. Condens. Matter 21, 345501 (2009).

${ }^{42}$ J. J. Rehr and A. Ankudinov, Int. J. Quantum Chem. 95, 487 (2003).

${ }^{43} \mathrm{P}$. Krüger (private communication).

${ }^{44}$ F. M. F. de Groot, J. C. Fuggle, B. T. Thole, and G. A. Sawatzky, Phys. Rev. B 42, 5459 (1990).

${ }^{45}$ G. Fronzoni, M. Stener, P. Decleva, F. Wang, T. Ziegler, E. van Lenthe, and E. Baerends, Chem. Phys. Lett. 416, 56 (2005).

${ }^{46}$ G. Fronzoni, M. Stener, P. Decleva, M. d. Simone, M. Coreno, P. Franceschi, C. Furlani, and K. C. Prince, J. Phys. Chem. A 113, 2914 (2009). 